\title{
Motivated to Retrieve: How Often Are You Willing to Go Back to the Well When the Well Is Dry?
}

\author{
Michael R. Dougherty and J. Isaiah Harbison \\ University of Maryland
}

\begin{abstract}
Despite the necessity of the decision to terminate memory search in many real-world memory tasks, little experimental work has investigated the underlying processes. In this study, the authors investigated termination decisions in free recall by providing participants an open-ended retrieval interval and requiring them to press a stop button when they had finished retrieving. Three variables important to assessing one's willingness to search memory were examined: (a) the time spent searching memory after the last successful retrieval before choosing to quit (the exit latency); (b) task difficulty; and (c) individual differences in motivation, as measured by Webster and Kruglanski's (1994) Need for Closure Scale. A strong negative correlation was found between individual differences in motivation and participants' exit latencies. This negative correlation was present only when the retrieval task started out as relatively difficult.
\end{abstract}

Keywords: recall, memory retrieval, exit latency, stopping rules

A fundamental component of memory retrieval is the decision to terminate search. This decision process characterizes many realworld memory retrieval tasks, from the generation of clinical diagnoses by physicians to the recollection of items from a grocery list left at home. Despite the necessity of the decision to terminate memory search in virtually all real-world tasks, little experimental work has investigated the underlying processes.

Related to the issue of search termination is the question of what psychological factors affect one's willingness to continue to search memory. Some factors are anticipated by models of memory, such as the use of familiarity in paired-associate retrieval (Diller, Nobel, \& Shiffrin, 2001) or feelings of knowing when searching for a question's answer (Barnes, Nelson, Dunlosky, Mazzoni, \& Narens, 1999; Costermans, Lories, \& Ansay, 1992; Nelson, Gerler, \& Narens, 1984; Young, 2004). However, other variables likely are important, too. For example, you presumably would search memory longer for a relatively important task, such as retrieving your grandmother's medications upon admitting her for emergency care. In contrast, search might be truncated relatively quickly for a comparatively unimportant task, such as recollecting movies you recently watched to provide a recommendation for a friend. Variables such as the importance of the retrieval task and idiographic levels of motivation likely serve as important moderators in the process of determining the amount of time one is willing to search memory.

Michael R. Dougherty and J. Isaiah Harbison, Department of Psychology, University of Maryland.

This material is based on work supported by the National Science Foundation under Grant SES-0134678, awarded to Michael R. Dougherty. Order of authorship is arbitrary. The authors thank Eddy Davelaar and Rick Thomas for the many stimulating discussions surrounding this project and Melanie Reyer and Avi Wershbale for assisting in data collection.

Correspondence concerning this article should be addressed to Michael R. Dougherty, Department of Psychology, University of Maryland, College Park, MD 20740. E-mail: mdougherty@psyc.umd.edu
In this article, we ask how long people will continue going back to the well (i.e., memory) when the well is dry (i.e., when retrieval has been unsuccessful). In addressing this question, we examined three variables important to assessing one's willingness to search memory: (a) the time spent searching memory after the last successful retrieval before choosing to quit; (b) task difficulty; and (c) individual differences in motivation, as measured by Webster and Kruglanski's (1994) Need for Closure (NFC) Scale. We hypothesized that individual differences in motivation would predict how long participants would spend searching memory in the absence of successful retrieval.

\section{Motivation and the Decision to Terminate Search}

The decision to terminate search arguably is as important as is the decision to initiate search, for two reasons. First, termination decisions can affect the number of items successfully retrieved, depending on when one decides to terminate search. Second, termination decisions imply costs (Anderson \& Milson, 1989), in that the number of elements retrieved from memory is traded off against time in task and mental effort, not to mention the possible economic consequences (e.g., the costs of truncating search prematurely may be relatively high within the context of medical diagnosis). For example, one is likely to be more persistent in searching memory if the expected gain of continuing memory search outweighs the expected costs.

Persistence, or an unwillingness to stop performing a task, is central to the construct of motivation (Ryan \& Deci, 2000). Within memory research, motivation is assumed to affect how much time or effort one devotes to the storage and maintenance of information, which ultimately has concomitant effects on retrieval (Cuvo, 1974; Eysenck \& Eysenck, 1980; Weiner, 1966; Wickens \& Simpson, 1968). For example, a student interested in chemistry is likely to spend more time studying the subject (encoding) and more time thinking about what she has learned about it (rehearsing). How- 
ever, few studies have documented the role of motivation at retrieval.

One reason for the apparent failure to document a relationship between motivation and memory at retrieval may be due to the fact that prior research has used, almost exclusively, fixed retrieval intervals for free-recall tasks. Giving participants a fixed retrieval interval eliminates (experimentally) any effect that individual differences in persistence might have on the duration of memory search. Indeed, in a study that used a paired-associates task with an open-ended response window, Loftus and Wickens (1970) showed that manipulations of motivation (e.g., incentives) increased time spent searching memory.

As mentioned above, traditional memory paradigms typically employ fixed retrieval intervals, in which participants are given a specific amount of time (e.g., $2 \mathrm{~min}$ ) to retrieve from memory. The top panel of Figure 1 presents a graphical depiction of this type of design. A common dependent variable within this paradigm is the interretrieval times (IRTs), defined as the latencies between successive retrievals (Rohrer \& Wixted, 1994). IRTs provide insight into the underlying dynamics of memory retrieval processes. One limitation of the fixed-retrieval-interval design is that it does not allow the researcher to measure how long the participant takes before terminating memory search.

The fixed-retrieval-interval paradigm can be modified to provide an open-ended response window that allows participants to set their own retrieval intervals, as depicted in the bottom half of Figure 1. When participants are given control over search termination, two additional temporal variables become meaningful: (a)

Experimenter Allotted Time for Retrieval
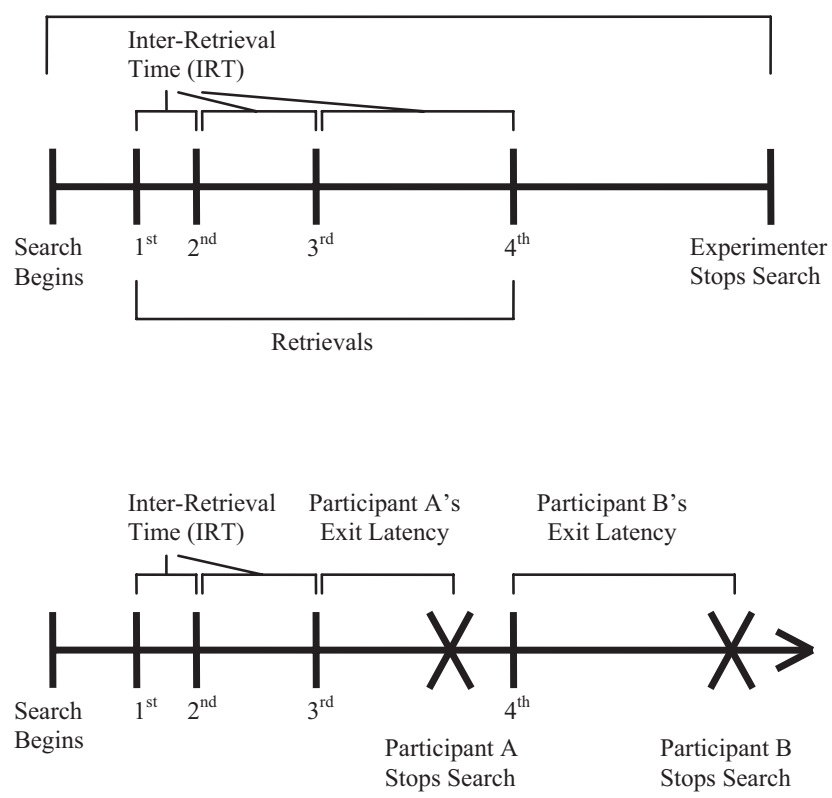

Figure 1. Representation of the retrieval process. The top panel shows the standard experimental design for multiple-item retrieval from memory. Here, the retrieval interval is determined by the experimenter, and the interretrieval times are often measured. The bottom panel shows the design used in the present experiment. Here, the participant determines the retrieval interval. The $X$ s demark the spots where two hypothetical participants might choose to terminate search. IRT $=$ interretrieval time. the total time spent in search and (b) the latency between the last successful retrieval and the decision to terminate search. We refer to the latter variable as the exit latency. ${ }^{1}$ The $X \mathrm{~s}$ in Figure 1 depict the stopping points of two hypothetical participants, denoted A and B. As is the case for Participants A and B, the total time spent in search and in the exit latencies likely differs across individuals and reflects one's personal rule for choosing when to terminate search.

Both total time and exit latencies relate to how long people spend searching memory. Total time is a function of both the number of successful retrieval attempts and the amount of time spent searching without success. Obviously, total time will increase monotonically with the number of items retrieved, meaning that total time confounds number of items retrieved with exit latencies. In contrast, exit latencies measure how long people continue to search memory in the absence of an additional successful retrieval. In other words, it measures how willing people are to continue searching memory when they appear to have exhausted the search space. Thus, exit latency is not confounded with the number of items retrieved and therefore serves as a purer measure of an individual's willingness to continue searching memory. Moreover, given the negatively accelerated function relating cumulative recall to time (Bousfield \& Sedgewick, 1944), relatively large differences in participants' willingness to continue memory search without successful retrieval might yield relatively small differences in the total number of items retrieved. Thus, exit latencies should also be a more sensitive measure of the willingness to continue memory search than is the number of items retrieved.

In the present study, control of when to terminate search was given to participants. This modification made it possible for us to capture the degree to which motivation, in particular persistence, influences memory search processes. In much the same way that persistence influences how much time one spends studying, we suggest, persistence is likely to influence how much time is spent attempting to retrieve information from memory. Given this as the case, providing participants with a fixed retrieval interval masks a variable critical for assessing the role of motivation on memory retrieval, namely, how long they are willing to continue searching memory before deciding that they can retrieve nothing more.

We measured individual differences in motivation using the Need for Closure (NFC) Scale (Webster \& Kruglanski, 1994). The NFC Scale includes subscales that measure two main aspects of how people acquire and use information. We were concerned with the Decisiveness subscale (see the Appendix), as it is assumed to measure how quickly people are willing to adopt a solution to a problem and is negatively correlated with how long participants search for information in the environment before making a decision (Kruglanski \& Webster, 1996; Neuberg, Judice, \& West, 1997). As this subscale appears to measure persistence in information search, we predicted that it would show a negative correlation with exit latency: More decisive participants should truncate memory search more quickly.

\footnotetext{
${ }^{1}$ By way of preview, we use the term exit latency to refer to the raw latency between the final item retrieved and the decision to terminate search. The term exit rate refers to the rate transformation of the exit latencies (1/exit latency). All statistical analyses were performed using the exit rate.
} 
NFC, as well as the decisiveness subcomponent, varies between individuals as well as within individuals across different tasks (Kruglanski \& Webster, 1996). Tasks that require more processing effort increase an individual's NFC. For this reason, we manipulated task difficulty by having participants retrieve high- or lowfrequency words. By several objective criteria, low-frequency words are ostensibly more difficult to retrieve: Participants respond more slowly to and generate fewer low-frequency words than they do high-frequency words (Delosh \& McDaniel, 1996; Hall, 1954; Landauer \& Freedman, 1968; McDaniel, DeLosh, \& Merritt, 2000; Watkins, LeCompte, \& Kim, 2000). Although all participants in our experiment were given blocks of high- and low-frequency words, the order of these blocks was counterbalanced. This feature allowed us to examine whether the hypothesized relationship between decisiveness and exit latency depended on the difficulty of the particular list or if the perceived difficulty of the task, as instantiated on the initial lists, established how decisiveness related to exit latencies. If the role of decisiveness is dependent on the difficulty of the particular list being retrieved, we would expect decisiveness to correlate with exit latencies for low-frequency lists, regardless of whether the low-frequency lists appeared in the initial blocks or after the high-frequency blocks. However, if decisiveness were initiated on the basis of the perceived difficulty of the task, we would expect the correlation between decisiveness and exit latency to manifest for both lowand high-frequency lists but only for participants whose initial lists consisted of the difficult, low-frequency lists. Note that although we focused on word frequency in our experiment, the underlying variable of interest was the actual or perceived difficulty of the retrieval task. Thus, we assumed other variables that affect difficulty of retrieval would have a similar effect on the role of decisiveness in recall.

As a final variable of interest, the counting-span (c-span) task was included as an individual-difference measure of working memory (WM) capacity. Prior research has found that WM capacity (or attentional control) is important in retrieval tasks that entail inhibition (Rosen \& Engle, 1997, 1998). For example, Rosen and Engle (1997) argued that recall differences between high- and low-span participants were due to high-span participants' better ability to inhibit the sampling and/or output of previously retrieved items. Our purpose for including a measure of WM was to test for the possible moderating role of WM in participants' decisions to terminate search. We reasoned that the tendency to resample previously retrieved items might serve as a cue that the search space has been exhausted. Inasmuch as low-span participants have a greater tendency to resample, they might also be quicker to terminate search in the absence of being able to retrieve new items. A second reason for including measures of WM is that we wanted to determine whether any observed relationship between decisiveness and exit latency could be accounted for by individual differences in WM capacity.

\section{Method}

\section{Design and Procedure}

Stimuli consisted of a total of 140 high-frequency words and 140 low-frequency words drawn from the MRC Psycholinguistic Database (Wilson, 1988; available from http://www.psy .uwa.edu.au/mrcdatabase/uwa_mrc.htm). The average frequency for low-frequency words was 2.34, and the average frequency for high-frequency words was 289.84 (Kucera \& Francis, 1967). Six low-frequency word lists and six high-frequency word lists were constructed for each participant. Each list consisted of 10 target items and a cue item and was constructed randomly for the participant. The experimental design was a $2 \times 2$ mixed factorial, with word frequency manipulated within subjects and order (highvs. low-frequency words presented first) counterbalanced across subjects.

Seventy-two University of Maryland psychology students were randomly assigned to one of two conditions. In the difficult-first condition, participants were given the low-frequency lists first. After they had completed two blocks of low-frequency lists, these participants were given the high-frequency lists. Participants in the easy-first condition received the blocks in the opposite order.

The memory task consisted of four blocks. Within each block, participants were first shown the cue word, which was unique to each list, along with the 10 target words contained on that list. The target words appeared with the cue word one at a time, consecutively, for $4 \mathrm{~s}$ each, until all of the target words for the first list had been shown. Then, participants were shown the second cue word paired with each of the target words, followed by the third cue word and target words. After the presentation of all the items on the third list, all three lists were shown a second time in exactly the same order as before, including the order of the target words. Following the second presentation of the three lists, participants began the recall portion of the block.

During recall, participants were presented with a single cue word in the middle of the screen and were asked to retrieve as many words as they could that had been previously studied with the cue. Participants were free to retrieve the words associated with the given cue in any order. Responses were made by speaking into a voice-key-activated microphone. Participants were told, "When you cannot remember any additional words, press the spacebar." Ten items were associated with each cue; therefore, participants could potentially generate a total of 10 correct items. The experimental software recorded up to 10 total responses.

This procedure was repeated for each cue word within each block, with participants completing a total of four blocks. For the difficult-first condition, the first two blocks were of low-frequency words and the second two blocks were of high-frequency words. This order was reversed for the easy-first condition.

Interitem retrieval times and exit latencies were extracted by analyzing the resulting audio files. Separate .wav audio files were created each time the participant made a sound loud enough to register with or to trigger the experimental program. In addition, the response time or the time of each trigger was recorded in a data file. The audio files sometimes included false alarms (e.g., a participant saying "uh" between responses) or misses (e.g., a participant speaking too softly to trigger the program). Each audio file was checked by listening to and visually inspecting it in an audio editing program. The content of the sound file (i.e., the word spoken) was recorded, and any necessary corrections to the response time data file were made.

After completing the fourth and final block, participants completed the NFC Scale, followed by the c-span task (Kane et al., 2004), as a measure of individual differences in WM capacity. The c-span task was performed individually in the presence of an 
experimenter. In the task, the participant was presented a screen with one to nine dark blue circles and an unrelated number of light blue circles and dark blue squares. The participant counted aloud each dark blue circle on the screen, one at a time. When the participant finished counting the dark blue circles and repeated their total number, the next screen was presented by the experimenter. This process was repeated for 2 to 6 screens. After the final screen, the participant had to recall the number of dark blue circles on each previous screen in serial order. An "X," or null response, could be entered for the number on a screen that was not remembered. The c-span score calculated for each participant was the total number of correct responses in the correct serial position over the 15 screens.

\section{Results}

The key dependent variables were the decisiveness scores, number of words retrieved, the total time spent retrieving, and the exit latencies. All statistical analyses on total time and exit latencies used the inverse (i.e., rate) transformation (e.g., 1/total time, 1/exit latency). The term exit rate is used to refer to these transformed exit latencies, and total rate is used for the transformed total time (for justification of using inverse transformation on latencies, see Ratcliff, 1993). Note that under the inverse transformation, large latencies are indexed by small rates.

Consistent with previous research and the characterization of the low-frequency lists as more difficult, participants in both conditions generated more high-frequency words than they did lowfrequency words. There was a main effect for word frequency, $F(1$, $70)=93.820, p<.001$, but this effect was qualified by a significant condition for word-frequency interaction, $F(1,70)=$ 9.188, $p<.01$. As shown in Figure 2, the mean number of words recalled from the two low-frequency lists was approximately the same for the two conditions, whereas participants retrieved fewer high-frequency words in the easy-first condition compared with the difficult-first condition. The between-subjects comparison of

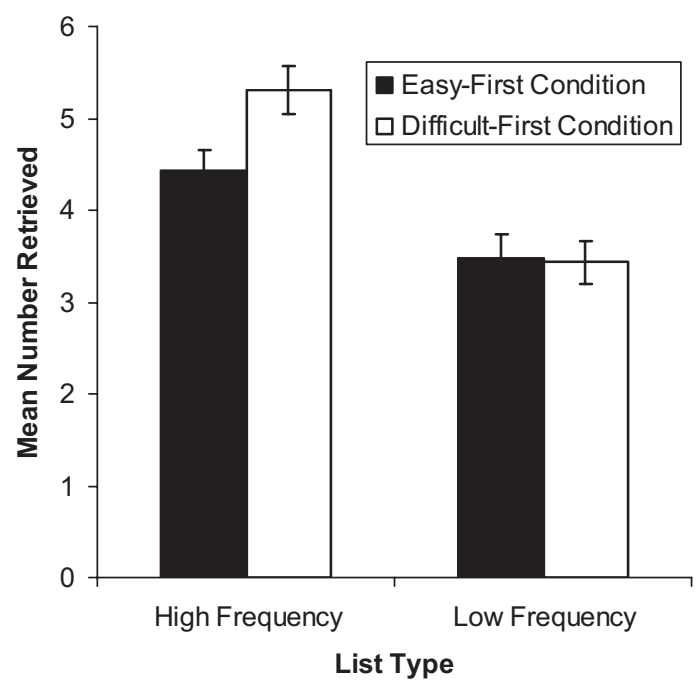

Figure 2. Mean number retrieved by condition (easy-first or difficultfirst) and by list type (high frequency or low frequency). Error bars represent standard errors of the means. high- versus low-word frequency was also significantly different when we compared only the first set of lists for each condition, $t(70)=3.074, p<.01$.

\section{Motivation and Memory Search}

We predicted a positive relationship between the speed of search termination and decisiveness, with more decisive individuals expected to terminate retrieval more quickly (i.e., to have larger exit rates) than less decisive individuals. However, we also expected the correlations to depend on the difficulty of the individual items being retrieved and/or the perceived difficulty of the retrieval task in general. For example, if the relationship between decisiveness and speed of termination is specific to those items that are difficult to retrieve, we would expect a correlation between decisiveness and exit rate to manifest for low-frequency lists, regardless of whether the low-frequency lists came before or after the highfrequency lists. In contrast, if the relationship between decisiveness and retrieval is sensitive to the perceived difficulty of the retrieval task in general, we would expect a carryover effect, wherein decisiveness and exit rates would be correlated for both low- and high-frequency lists but only for participants who had received the low-frequency lists first. We assessed this prediction by testing the relationship between decisiveness and both total time spent in search and exit latency.

Table 1 provides the overall correlations between decisiveness and the temporal variables of interest, as well as other temporal variables. Note that the correlations are separated by list type (high vs. low frequency). Therefore, the estimates for each participant were the average over six high-frequency lists and the average over six low-frequency lists. We include the other temporal variables (the rate of retrieving the first and second items) to determine if decisiveness was correlated with all the temporal variables or just the ones of interest. As shown in Table 1, these other variables were not significantly correlated with decisiveness.

The correlations between decisiveness and total rate (1/total time) were significantly positive for both the high- and lowfrequency lists in the difficult-first condition. The more decisive participants were, the less time they spent in the task. The same correlations were virtually zero in the easy-first condition. However, as indicated above, we believe that motivation plays its role after the final successful retrieval. If this is the case, the relationship between total rate and decisiveness is due to the exit latency's inclusion in the total time measure. Indeed, when the contribution of exit rate is partialled out, the relationship between total rate and decisiveness is no longer significant in the difficult-first condition for either the high-frequency lists $(r=-0.183)$ or the lowfrequency lists $(r=.209)$.

As expected, the relationship between exit rate and decisiveness was the same as that between total rate and decisiveness. Exit rate and decisiveness had a significantly positive correlation for both high- and low-frequency words in the difficult-first condition. As with total rate, these same correlations in the easy-first condition were virtually zero.

One finding of particular importance was that the exit rates were correlated with the number of items retrieved. That is, within subjects, the more items an individual retrieved for a particular list, the quicker he or she was to terminate search on that list. This finding, which we elaborate on below, was problematic for inter- 
Table 1

Pearson $r$ Correlations Between Decisiveness and the Main Variables of Interest

\begin{tabular}{lcc}
\hline \multicolumn{1}{c}{ Condition } & $\begin{array}{c}\text { Low-frequency list } \\
r\end{array}$ & $\begin{array}{c}\text { High-frequency list } \\
r\end{array}$ \\
\hline & \multicolumn{2}{c}{$r$ (Decisiveness, exit rate) } \\
Difficult first $(n=34)$ & $.576^{* * *}$ & $.666^{* *}$ \\
Easy first $(n=38)$ & .118 & .009 \\
\hline & & \\
& $r$ (Decisiveness, rate of generating first) \\
Difficult first $(n=34)$ & .190 & .128 \\
Easy first $(n=38)$ & -.024 & .161 \\
\hline
\end{tabular}

\begin{tabular}{lcc} 
& $r$ (Decisiveness, rate of generating second) \\
Difficult first $(n=34)$ & -.002 & -.003 \\
Easy first $(n=38)$ & .072 & -.024 \\
\hline
\end{tabular}

\begin{tabular}{lcc} 
& $r$ (Decisiveness, total time $)$ \\
Difficult first $(n=34)$ & $.488^{* *}$ & $.367^{*}$ \\
Easy first $(n=38)$ & -.099 & .087 \\
\hline
\end{tabular}

\begin{tabular}{lrr} 
& \multicolumn{2}{c}{$r($ WM span, exit rate) } \\
Difficult first $(n=33)$ & .171 & -.083 \\
Easy first $(n=38)$ & .117 & -.059
\end{tabular}

Note. $\mathrm{WM}=$ working memory.

${ }^{*} p<.05 .{ }^{* *} p<.01$.

pretation of our overall correlations, because participants varied on how many items they retrieved for the high- and low-frequency lists. To ensure that the overall correlations were not due to differences between conditions in the total number of items retrieved, we examined the correlation between decisiveness and exit rate, controlling for the number retrieved. For these analyses, we separated each participant's data by the number of words retrieved and took the average exit rate for each set size. We then plotted participants' exit rate by decisiveness score separately for each set size. These plots are presented in Figures 3 and 4, along with the best fitting linear regression line.

Only a subset of the participants contributed to each graph, which resulted in an unequal number of participants in each graph. Nevertheless, these individual analyses are quite informative. As can be seen in Figures $3 \mathrm{~A}$ and $3 \mathrm{~B}$, the correlations between decisiveness and exit rate in the difficult-first condition were positive in all 12 cases. The probability of obtaining 12 out of 12 correlations in the predicted direction by chance is $p<.001$, by sign test. In contrast, in the easy-first condition, shown in Figures $4 \mathrm{~A}$ and $4 \mathrm{~B}$, the correlations were positive in only 5 of the 12 cases. Thus, individual differences in decisiveness appear to consistently predict how long participants will be willing to continue searching memory before giving up but only when the task set is perceived as sufficiently challenging. Note that the key variable is not word frequency per se, as the relationship between decisiveness and exit latency was nearly zero when the low-frequency words occurred after the high-frequency lists. Thus, we argue that the role of decisiveness in the decision to terminate memory search is set by the initial difficulty of the task and is then carried forth throughout the experiment.

\section{Exit Latency, WM Span, and Number Retrieved}

The above analyses establish the relationship between decisiveness and the exit latency. However, as suggested in the introduction, other variables may be important as well. Two such variables that might be related to the exit latency are individual differences in WM and the size of the set of retrieved items (or, conversely, the number of items remaining in the set).

Exit latency and WM span. As indicated previously, prior work has shown a positive relationship between individual differences in WM span and the number of items retrieved when the retrieval task requires inhibition (Rosen \& Engle, 1997, 1998). One possible cue to terminate the retrieval process is the number of times one resamples already retrieved items (Shiffrin, 1970). Inasmuch as WM span is negatively correlated with the likelihood of inhibiting previously retrieved items, low-span participants might also be prone to exit memory search more quickly. Our main questions were (a) are measures of motivation independent of measures of WM and (b), if so, do individual differences in WM span correlate with decisions to terminate search.

First, WM span was not correlated with decisiveness, either within condition or collapsing across condition (all $p \mathrm{~s}>.20$ ). More important, WM span did not predict exit latencies (all $p \mathrm{~s}>$ .20; see Table 1). Needless to say, the correlation between decisiveness and exit rate remained unchanged ( $r=.68$ for highfrequency lists and $r=.60$ for low-frequency lists), even after partialling out the variance due to WM span.

Exit latency and number retrieved. Another potential cue for terminating memory search is the number of items retrieved. To address whether decisions to terminate search were sensitive to the number of items retrieved, we computed within-subject gamma correlations between the total number of items retrieved for each list and their corresponding exit rates. The mean gamma correlations are shown in Figure 5. As can be seen, there was a significant positive relationship between exit rates and the number retrieved for all four conditions. Thus, the more items a participant retrieved, the more quickly he or she terminated memory search following the final retrieval.

The relationship between exit rate and number retrieved, shown in Figure 5, appears to vary across conditions and list types. The interaction between list type and condition was significant, $F(1$, $70)=8.792, p<.01$. There was no main effect for word frequency, $F(1,70)<1$. The magnitude of the gamma correlations increased from the first set of lists to the second set of lists. That is, the exit rate-number retrieved relationship was stronger for the second set of lists than it was for the first, regardless of whether participants saw the low-frequency list or the high-frequency list first.

\section{Discussion}

Our purpose in this experiment was to examine the relationship between motivation and memory retrieval processes. In so doing, this study makes several novel contributions to the literature on memory retrieval. Methodologically, the present research demonstrates a design in which decisions to terminate memory search can be measured. Rather than providing participants with a closed response window, we provided participants with an open-ended response window and allowed them to terminate the retrieval 
A Difficult-First: High Frequency Lists
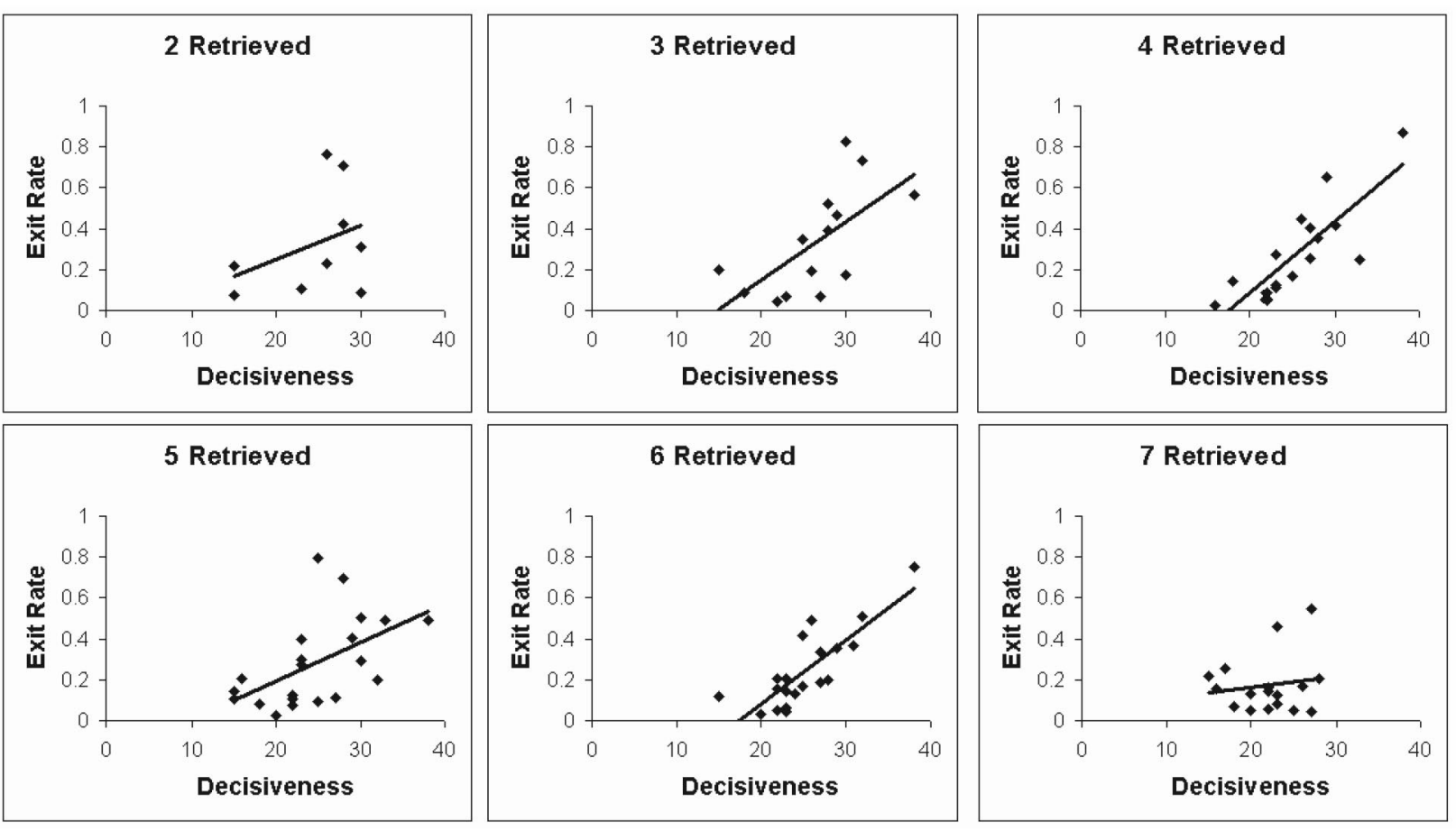

\section{B Difficult-First: Low Frequency Lists}
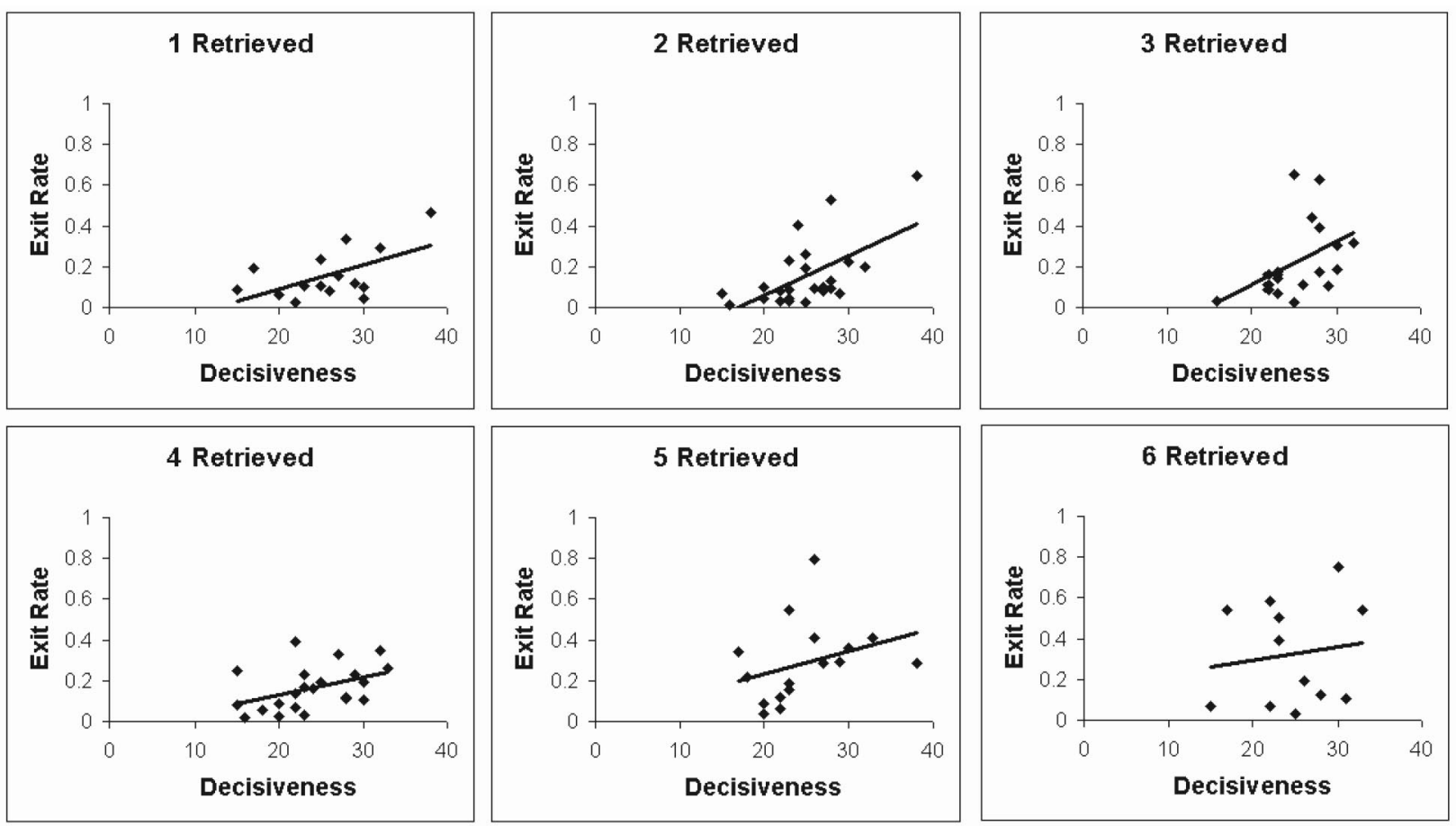

Figure 3. Exit rate (1/exit latency) and decisiveness by number retrieved for the difficult-first condition. The relationship between exit rate and decisiveness is shown separately for each total number of items retrieved, for the high frequency lists in Panel A and the low frequency lists in Panel B. 
A Easy-First: High Frequency Lists
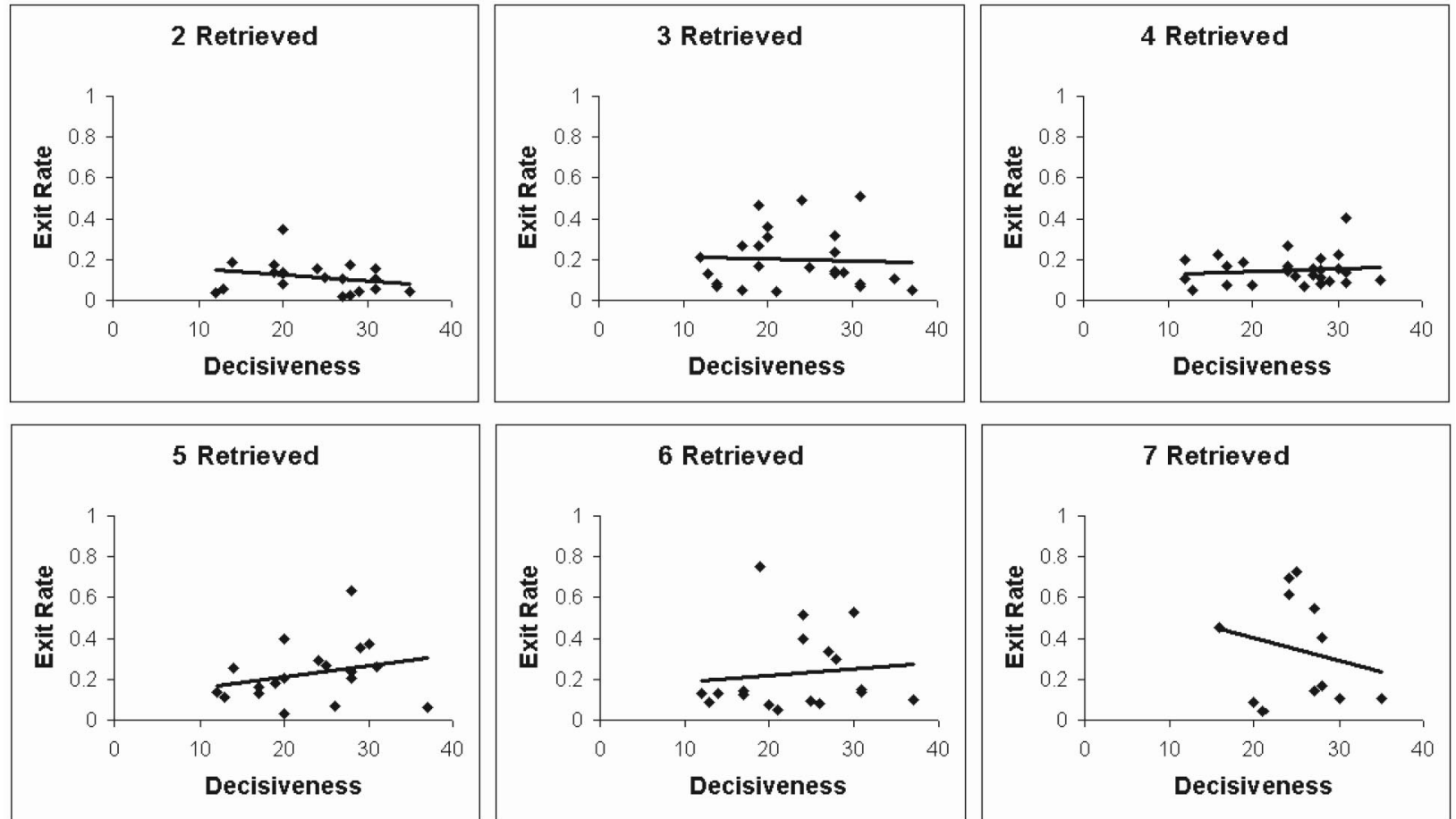

\section{B Easy-First: Low Frequency Lists}
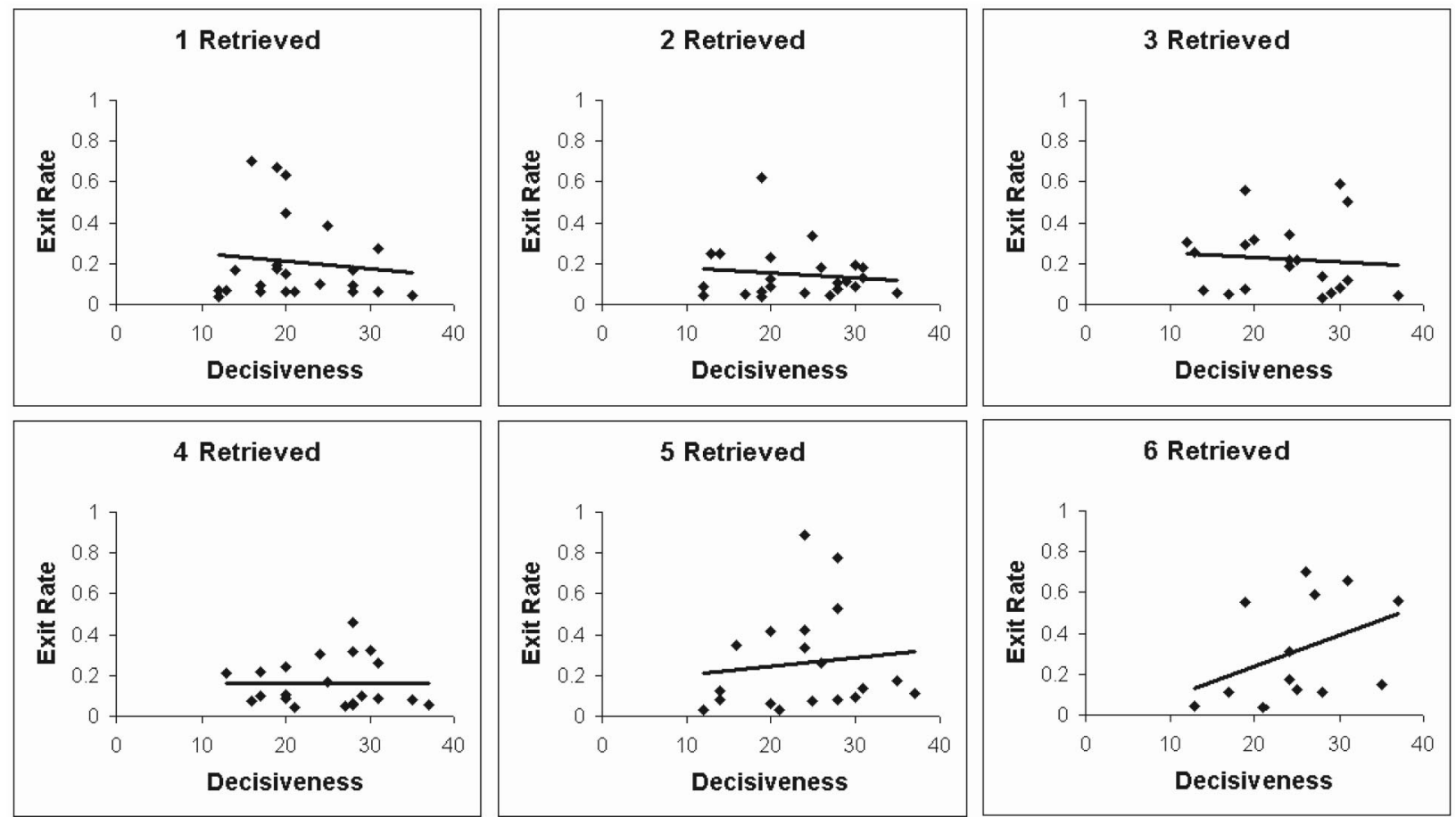

Figure 4. Exit rate (1/exit latency) and decisiveness by number retrieved for the easy-first condition. The relationship between exit rate and decisiveness is shown separately for each total number of items retrieved, for the high-frequency lists in Panel A and the low-frequency lists in Panel B. 


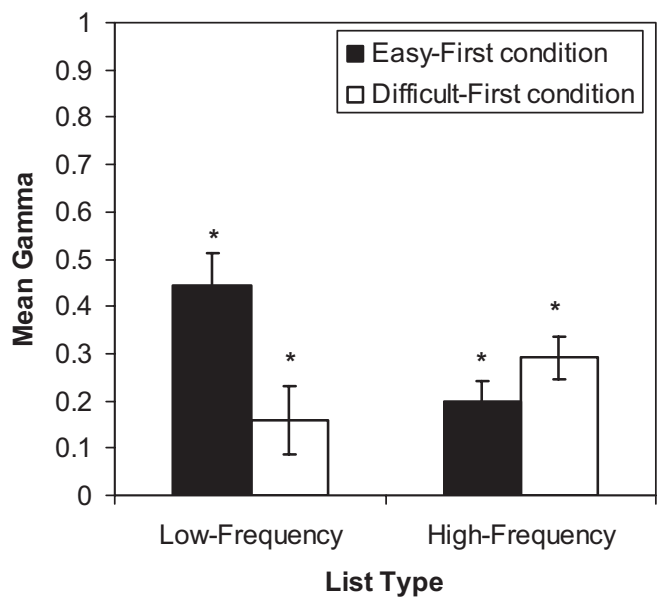

Figure 5. Mean within-subject gammas for exit rate and number of items recalled for both the high- and low-frequency lists, for both the difficultfirst and easy-first conditions. Error bars represent standard errors of the means. ${ }^{*} p \leq .05$ (significant from zero by one sample $t$ test).

process on their own. The design enables the measurement of both the total amount of time a participant is willing to search and, more important, the time between retrieval of the final item and the decision to terminate retrieval. These temporal data are fundamental both for understanding the factors that affect how people terminate retrieval and for assessing the validity of stopping rules that have been implemented in computational models of memory (e.g., ACT-R, Anderson, Bothell, Lebiere, \& Matessa, 1998; SAM, Raaijmakers \& Shiffrin, 1981; REM, Shiffrin \& Steyvers, 1997).

Empirically, our research has provided several noteworthy findings. First, we demonstrated that an individual difference variable is an important moderator of retrieval processes: Exit latency was related to individual differences in decisiveness. This finding fits nicely with research on external information search, in which participants high in decisiveness tend to terminate information search more quickly than do participants low in decisiveness (Kruglanski \& Webster, 1996). Our research extends this finding to the internal information-search process of memory retrieval.

A second but related finding was that the relationship between exit latency and decisiveness appears to be moderated by taskrelated variables, namely, the difficulty of the retrieval task. $\mathrm{Cu}$ riously, the decisiveness and exit-latency correlation was not related to list-specific difficulty but appears to manifest on the basis of the difficulty of the initial tasks. The relationship between decisiveness and exit latency was present only when participants received the more difficult, low-frequency lists first. This relationship then carried over to the easier, high-frequency lists. If participants received the easier lists first, no evidence was found for the relationship between decisiveness and exit latency for either the more difficult or the easier lists.

A third important finding in our research was that exit latencies decreased as a function of the total number of items retrieved. This finding is important, because it constrains the set of plausible stopping rules to those that predict that exit latency is a decreasing function of number of items retrieved.
One particularly interesting stopping rule was proposed by Anderson and Milson (1989; see also Anderson \& Schooler, 1991), who suggested that termination decisions should be a function of three factors: the cost $(\mathrm{C})$ of retrieving an item, the gain $(\mathrm{G})$ or benefit from retrieving the item, and the probability $(\mathrm{P})$ that an item will be needed in the current context. Assuming that $\mathrm{C}$ is a function of the time and effort required to retrieve an item and that the amount of effort is constant across time, the cost of retrieval can be considered equivalent to the time spent in retrieval. The stopping rule for memory search is $\mathrm{C}>\mathrm{PG}$, where $\mathrm{PG}$ is the cost threshold, or expected gain. When $\mathrm{C}$ exceeds $\mathrm{PG}$, retrieval is terminated. Note that $\mathrm{P}$ measures how likely an item is to be needed, on the basis both of the general history of occurrence and of occurrence in the specific context.

$\mathrm{P}$ is of particular importance to the current discussion, because it is assumed to determine the order of retrieval and each item's retrieval latency (Anderson \& Milson, 1989). Items with a larger P are retrieved earlier in serial order and more quickly than are items with a smaller P. Variations in P predict the observed relationship between exit latency and number of items retrieved. As more items are retrieved, $\mathrm{P}$ decreases. As $\mathrm{P}$ decreases, retrieval gets more costly (takes more time) and at the same time reduces PG, the threshold of allowable cost. As the cost threshold decreases, so should the amount of time (or cost) spent retrieving before search is terminated. Therefore, $\mathrm{C}>\mathrm{PG}$ anticipates that exit latencies will be shorter as the number of items retrieved increases.

However, we also found that the relationship between the exit rate and number retrieved was stronger for the second set of blocks, for both the easy-first and the difficult-first condition. It could be that increased experience in the task affects study strategy, as strategy is expected to affect $\mathrm{P}$ by changing the input into memory (Anderson \& Milson, 1989). Still, absent a theory of strategy selection, it is not clear why we would expect an increase rather than a decrease in the relationship between exit rate and number retrieved as participants continued the task. Furthermore, the difference in the effect of decisiveness between conditions would require a more developed theory. Though the stopping rule includes a variable that could be linked with motivation $(G)$, why $\mathrm{G}$ would play a role in the difficult-first condition but not the easy-first condition is unclear.

The difficulty in accounting for the exit latency data and the relationship of exit latency with decisiveness suggests that much more theoretical work on stopping rules is needed. Indeed, even the most developed stopping rule, that used by Anderson and Milson (1989), fails to easily account for the full pattern of results. On the other hand, the relationship between motivation (specifically, decisiveness) and exit latencies seems intuitive; one would expect highly motivated participants to spend more time searching for information (Loftus \& Wickens, 1970). This relationship between retrieval processes and motivation has proven elusive for two reasons. First, researchers have focused on number of items retrieved as the main dependent variable for measuring the effect of motivation. Second, the experimental paradigm typically used in memory research masks an important component of the retrieval process that may be related to motivation, namely, how long one persists in memory retrieval. In sum, the present research demonstrates a method of measuring the decision to terminate memory search and how individual differences might moderate basic retrieval processes. 


\section{References}

Anderson, J. R., Bothell, D., Lebiere, C., \& Matessa, M. (1998). An integrated theory of list memory. Journal of Memory and Language, 38, 341-380.

Anderson, J. R., \& Milson, R. (1989). Human memory: An adaptive perspective. Psychological Review, 96, 703-719.

Anderson, J. R., \& Schooler, L. J. (1991). Reflections of the environment in memory. Psychological Science, 2, 396-408.

Barnes, A. E., Nelson, T. O., Dunlosky, J., Mazzoni, G., \& Narens, L. (1999). An integrative system of metamemory components involved in retrieval. In D. Gopher \& A. Koriat (Eds.), Attention and Performance XVII: Cognitive regulation of performance: Interaction of theory and application (pp. 287-313). Cambridge, MA: MIT Press.

Bousfield, W. A., \& Sedgewick, C. H. W. (1944). An analysis of sequences of restricted associative responses. Journal of General Psychology, 30, $149-165$.

Costermans, J., Lories, G., \& Ansay, C. (1992). Confidence level and feeling of knowing in question answering: The weight of inferential processes. Journal of Experimental Psychology: Learning, Memory, and Cognition, 18, 142-150.

Cuvo, A. J. (1974). Incentive level influence on overt rehearsal and free recall as a function of age. Journal of Experimental Child Psychology, $18,167-181$

Delosh, E. L., \& McDaniel, M. A. (1996). The role of order information in free recall: Application to the word-frequency effect. Journal of Experimental Psychology: Learning, Memory, and Cognition, 22, 1136-1146.

Diller, D. E., Nobel, P. A., \& Shiffrin, R. M. (2001). An ARC-REM model for accuracy and response time in recognition and recall. Journal of Experimental Psychology: Learning, Memory, and Cognition, 27, 414435

Eysenck, M. W., \& Eysenck, M. C. (1980). Effects of monetary incentives on rehearsal and on cued recall. Bulletin of the Psychonomic Society, 15, 245-247.

Hall, J. F. (1954). Learning as a function of word frequency. American Journal of Psychology, 67, 138-140.

Kane, M. J., Hambrick, D. Z., Tuholski, S. W., Wilhelm, O., Payne, T. W., \& Engle, R. W. (2004). The generality of working memory capacity: A latent-variable approach to verbal and visuospatial memory span and reasoning. Journal of Experimental Psychology: General, 133, 189217.

Kruglanski, A. W., \& Webster, D. M. (1996). Motivated closing of the mind: "Seizing" and "freezing." Psychological Review, 103, 263-283.

Kruglanski, A. W., Webster, D. M., \& Klem, A. (1993). Motivated resistance and openness to persuasion in the presence or absence of prior information. Journal of Personality and Social Psychology, 65(5), 861876

Kucera, H., \& Francis, W. N. (1967). Computational analysis of presentday American English. Providence, RI: Brown University Press.

Landauer, T., \& Freedman, J. (1968). Information retrieval from long-term memory: Category size and recognition time. Journal of Verbal Learning \& Verbal Behavior, 7, 291-295.

Loftus, G. R., \& Wickens, T. D. (1970). Effect of incentive on storage and retrieval processes. Journal of Experimental Psychology, 85, 141-147.

McDaniel, M. A., DeLosh, E. L., \& Merritt, P. S. (2000). Order information and retrieval distinctiveness: Recall of common versus bizarre material. Journal of Experimental Psychology: Learning, Memory, and Cognition, 26, 1045-1056

Nelson, T. O., Gerler, D., \& Narens, L. (1984). Accuracy of feeling-ofknowing judgments for predicting perceptual identification and relearning. Journal of Experimental Psychology: General, 113, 282-300.

Neuberg, S. L., Judice, T. N., \& West, S. G. (1997). What the Need for Closure Scale measures and what it does not: Toward differentiating among related epistemic motives. Journal of Personality and Social Psychology, 72, 1396-1412.

Raaijmakers, J. G. W., \& Shiffrin, R. M. (1981). Search of associative memory. Psychological Review, 88, 93-134.

Ratcliff, R. (1993). Methods for dealing with reaction time outliers. Psychological Bulletin, 114, 510-532.

Rohrer, D., \& Wixted, J. T. (1994). An analysis of latency and interresponse time in free recall. Memory \& Cognition, 22, 511-524.

Rosen, V. M., \& Engle, R. W. (1997). The role of working memory capacity in retrieval. Journal of Experimental Psychology: General, 126, 211-227.

Rosen, V. M., \& Engle, R. W. (1998). Working memory capacity and suppression. Journal of Memory and Language, 39, 418-436.

Ryan, R. M., \& Deci, E. L. (2000). Self-determination theory and the facilitation of intrinsic motivation, social development, and well-being. American Psychologist, 55, 68-78

Shiffrin, R. M. (1970). Memory search. In D. A. Norman (Ed.), Models of human memory (pp. 375-447). New York: Academic Press.

Shiffrin, R. M., \& Steyvers, M. (1997). A model for recognition memory: REM-retrieving effectively from memory. Psychonomic Bulletin \& Review, 4, 145-166.

Watkins, M. J., LeCompte, D. C., \& Kim, K. (2000). Role of study strategy in recall of mixed lists of common and rare words. Journal of Experimental Psychology: Learning, Memory, and Cognition, 26, 239-245.

Webster, D. M., \& Kruglanski, A. W. (1994). Individual differences in need for cognitive closure. Journal of Personality and Social Psychology, 67, 1049-1062.

Weiner, B. (1966). Motivation and memory. Psychological Monographs: General and Applied, 80, 1-22.

Wickens, D. D., \& Simpson, C. K. (1968). Trace cue position, motivation, and short-term memory. Journal of Experimental Psychology, 76, 282285.

Wilson, M. D. (1988). The MRC Psycholinguistic Database: Machine readable dictionary, Version 2. Behavioural Research Methods, Instruments and Computers, 20, 6-11.

Young, C. J. (2004). Contributions of metaknowledge to retrieval of natural categories in semantic memory. Journal of Experimental Psychology: Learning, Memory, and Cognition, 30, 909-916. 


\section{Appendix}

\section{Decisiveness Subscale of the Need for Closure Scale}

When I go shopping, I have difficulty deciding exactly what it is that I want.*

When faced with a problem I usually see the one best solution very quickly.

I tend to put off making important decisions until the last possible moment.*

I usually make important decisions quickly and confidently.

I would describe myself as indecisive.*

I tend to struggle with most decisions.*

When trying to solve a problem I often see so many possible options that it's confusing.*
Note. From "Motivated Resistance and Openness to Persuasion in the Presence or Absence of Prior Information," by A. W Kruglanski, D. M. Webster, and A. Klem, 1993, Journal of Personality and Social Psychology, 65(5), pp. 861-876. Copyright 1993 by the American Psychological Association.

Responses are made on a 6-point scale from 1 (strongly disagree) to 6 (strongly agree). An asterisk means that the item is reverse scored.

Received January 19, 2007 Revision received July 19, 2007

Accepted July 24, 2007

\section{Members of Underrepresented Groups: Reviewers for Journal Manuscripts Wanted}

If you are interested in reviewing manuscripts for APA journals, the APA Publications and Communications Board would like to invite your participation. Manuscript reviewers are vital to the publications process. As a reviewer, you would gain valuable experience in publishing. The P\&C Board is particularly interested in encouraging members of underrepresented groups to participate more in this process.

If you are interested in reviewing manuscripts, please write to the address below. Please note the following important points:

- To be selected as a reviewer, you must have published articles in peer-reviewed journals. The experience of publishing provides a reviewer with the basis for preparing a thorough, objective review.

- To be selected, it is critical to be a regular reader of the five to six empirical journals that are most central to the area or journal for which you would like to review. Current knowledge of recently published research provides a reviewer with the knowledge base to evaluate a new submission within the context of existing research.

- To select the appropriate reviewers for each manuscript, the editor needs detailed information. Please include with your letter your vita. In the letter, please identify which APA journal(s) you are interested in, and describe your area of expertise. Be as specific as possible. For example, "social psychology" is not sufficient-you would need to specify "social cognition" or "attitude change" as well.

- Reviewing a manuscript takes time (1-4 hours per manuscript reviewed). If you are selected to review a manuscript, be prepared to invest the necessary time to evaluate the manuscript thoroughly.

Write to Journals Office, American Psychological Association, 750 First Street, NE, Washington, DC 20002-4242. 